\title{
WiMAX System Simulation and Performance Analysis under the Influence of Jamming
}

\author{
Rakesh Jha, Hardik Patel, Upena D. Dalal, Wankhede A. Vishal \\ Department of Electronics Engineering, Sardar Vallabhbhai National Institute of Technology, Surat, India. \\ Email: \{jharakesh.45, wankhedeva\}@gmail.com, p.hardik@eced.svnit.ac.in, upena_dalal@yahoo.com
}

Received May 15 ${ }^{\text {th }}$, 2010; revised June 20 ${ }^{\text {th }}, 2010$; accepted July $7^{\text {th }}, 2010$.

\begin{abstract}
This paper presents simulation of WiMAX based system under jamming. The performance of the system was found out to greatly differ with the use of different jamming signals, allowing central areas to be identified, where system development should be focused on. In addition, from the basic theory point of view, rather surprising results were also found. This work should give a clear picture of how the studied WiMAX system performs under jamming as well as without jamming. The results show that some forms of interference degrade the performance of the system rapidly, thus the form of incoming jamming should be known and considered before deploying the system. Single carrier jamming and multi-carrier jamming are discussed here. The issues related to jamming and jamming reduction techniques are also covered. Jamming can destroy communication in the targeted area. Multi-carrier jamming is challenge in WiMAX because WiMAX is having OFDM based physical layer. Simulation is the main approach in this paper. OPNET MODELER 14.5 is the software used for the simulation purpose.
\end{abstract}

Keywords: Jamming, Multicarrier, WiMAX, OFDM

\section{Introduction}

IEEE 802.16 is the standard for WiMAX. WiMAX is also known as wireless broadband. IEEE 802.16d - 2004 is known as fixed WiMAX and IEEE 802.16e - 2005 is known as mobile WiMAX [1]. In wired networks physical layer threats are not important but in wireless air is used as medium so physical layer threats comes into picture [2]. In wireless jamming and scrambling are considered as physical layer threats. Mac layer threats are different than physical layer threats. Here simulation approach is used to see the performance of the IEEE 802.16e 2005 fixed NLOS (Non line of sight) system in jamming environment. Jamming is achieved by introducing a source of noise strong enough to significantly reduce the capacity of the WiMAX channel. The information and equipment required to perform jamming are not difficult to acquire. Resilience to jamming can be augmented by increasing the power of signals or increasing the bandwidth of signals via spreading techniques such as frequency hopping or direct sequence spread spectrum. The practical options include a more powerful WiMAX transmitter, a high gain WiMAX transmission antenna, or a high gain WiMAX receiving antenna [3]. It is easy to detect jamming in WiMAX Communications as it can be heard by the receiving equipment. Law enforcement can also be involved to stop jammers. Since jamming is fairly easy to detect and address, so it does not pose a significant impact on both the WIMAX users and systems. Single carrier jamming and multi-carrier jamming are considered here for simulation approach. Single carrier jamming is used to jam the particular band of frequencies. In single carrier jamming carrier frequency and bandwidth of the targeted system should be known. In multi-carrier jamming the frequencies of carriers of targeted system should be known. Simulation approach is easy compare to practical approach. The issues related to practical approach will be described in the later part.

\section{The Investigated Physical Layer}

The primary operation bands of WiMAX include frequencies 10-66 GHz, 2-11 GHz and license-exempt frequencies below $11 \mathrm{GHz}$ (primarily 5-6 GHz). According to these operation bands, WiMAX PHY defines five specifications for different operation scenarios. Among them, Wireless MAN-OFDM PHY is based on orthogonal frequency-division multiplexing (OFDM) technology and designed for NLOS operation in the frequency bands below $1 \mathrm{GHz}$ [4]. It is selected to be the air interface of 
the system under investigation in this paper. Denial of service is very similar to jamming but it is MAC layer threat [5].

At the transmitter side, the information data first undergoes channel coding composed of randomization, forward error correction (FEC), and interleaving. Randomizer uses a Linear Feedback Shift Register (LFSR) to scatter long data strings of zeros or ones. FEC concatenates an outer Reed-Solomon encoder with an inner rate compatible convolutional encoder. FEC helps to correct the errors in subcarriers to a certain limit. The interleaver takes two permutations to rearrange the subcarriers so that the burst errors are distributed more uniformly at the demodulation input. After channel coding, data bits are mapped and modulated onto the allocated subcarriers by QPSK, 16-QAM and 64-QAM modulation. Subsequently, data are transmitted by OFDM method. In the receiver side, all the procedures carried out in the transmitter side are implemented again but in a reverse direction. One OFDM symbol can be divided into two parts in time domain: the cyclic prefix (CP) time and the useful symbol time [6,7]. The CP locates in the beginning of the symbol and is a duplication of the tail of the useful symbol, which is introduced to mitigate the effect of multipath. In frequency domain, an OFDM symbol is composed of a series of subcarriers. In Wireless MAN-OFDM PHY, the number of subcarriers is 256. As per Figure 1, three types of subcarriers can be categorized: 192 data subcarriers carrying payload, 8 pilot subcarriers mainly for channel estimation, and 56 null subcarriers for guarding purpose. The pilot subcarriers distribute evenly among the data subcarriers. This is standard symbol in frequency domain.

Channel estimation is mandatory for the OFDM systems employing coherent detection. Comb type pilot channel estimation is capable of collecting instant information of the channel and therefore used in this research. The channel estimation for the payload subcarriers is achieved by interpolation, using the channel information obtained at the 8 pilot subcarriers. In this paper IEEE 802.16e-2005 is simulated under jamming. In Mobile WiMAX, the FFT size is scalable from 128 to 2,048. Here, when the available bandwidth increases, the FFT size is also increased such that the subcarrier spacing is always $10.94 \mathrm{kHz}$. This keeps the OFDM symbol dura-

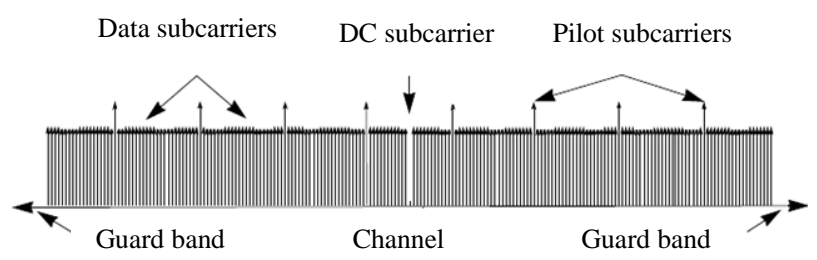

Figure 1. OFDM symbol frequency domain representation [7] tion, which is the basic resource unit, fixed and therefore makes scaling have minimal impact on higher layers. A scalable design also keeps the costs low. The subcarrier spacing of $10.94 \mathrm{kHz}$ was chosen as a good balance between satisfying the delay spread and Doppler spread requirements for operating in fixed and mobile environments. This subcarrier spacing can support delay-spread values up to 20 micro seconds and vehicular mobility up to $125 \mathrm{~km}$ per hour when operating in $3.5 \mathrm{GHz}$. A subcarrier spacing of $10.94 \mathrm{kHz}$ implies that 128, 512, 1,024, and 2,048 FFT are used when the channel bandwidth is $1.25 \mathrm{MHz}, 5 \mathrm{MHz}, 10 \mathrm{MHz}$, and $20 \mathrm{MHz}$, respectively. $2-11 \mathrm{GHz}$ is used for Fixed NLOS and 2-6 GHz is used for mobile NLOS. It should, however, be noted that mobile WiMAX may also include additional bandwidth profiles. For example, a profile compatible with WiBro will use an $8.75 \mathrm{MHz}$ channel bandwidth and 1,024 FFT. This obviously will require different subcarrier spacing and hence will not have the same scalability properties. The number of subcarriers may be 512, 1024 and 2048. Data subcarriers, Null subcarriers and Pilot subcarriers are also given in next section for 512, 1024 and 2048 subcarriers.

\section{Jamming in Detail}

There are two types of jamming: single carrier jamming and multicarrier jamming.

\subsection{Single Carrier Jamming}

The goal of single carrier jamming is to insert an interference signal into the enemy communication system so that the wanted signal is completely submerged by the interference. This form of jamming is also known as denial of service attack or obscuration jamming. The optimal jamming waveform is intuitively white Gaussian noise (WGN), since from the information theory point of view, it has maximum entropy. This conclusion can also be drawn from the fact that the receiver can not distinguish between jammer injected noise and its own [8-9]. Based on the relationship between jammer bandwidth and that of the equipment, single carrier jamming can be categorized into narrow- (spot) and wideband (barrage) jamming. The relationship is conveniently expressed as

$$
\frac{\mathrm{B}_{\mathrm{J}}}{\mathrm{B}_{\mathrm{Vs}}}=\frac{\text { Jammer_bandwidth }}{\text { Victim_system_bandwidth }}
$$

Typically, if the ratio BJ/BVS is less than 0.2 jamming is considered to be spot jamming and if greater than 1 , barrage jamming. The main advantage of single carrier jamming is that, very little information about the enemy's equipment is required. However, there are great many factors, which make the performance of a noise jammer to drop below its theoretical capability. The fact that a noise jammer has to function on victim systems using arbitrary polarizations, generally leads to usage of 
either 45 degrees slant polarized or circularly polarized jammer radiations. This causes a rather modest effective radiated power (ERP) drop of typically $3 \mathrm{~dB}$, but more serious losses in the order of tens of $\mathrm{dB}$ occur as a result of bad noise quality and e.g. orthogonal polarization between jammer and victim antennas. The easiest way of creating an effective noise jammer is to pass band-limited noise through an RF-amplifier and to the transmitting antenna. This method is also known as direct noise amplification (DINA).

\subsection{Multicarrier Jamming}

Multicarrier jamming differs from single carrier jamming by being suitable only for jamming the system it is designed for. The general idea is to determine the most critical vulnerability of the victim system in terms of the carriers used and then inject a very narrowband signal, e.g. zero bandwidth sine signal, onto the those carriers. If data subcarriers are destroyed by jamming then information is lost so throughput is reduced so bit error rate (BER) is increased. If pilot subcarriers are destroyed by jamming then channel estimation is very difficult. More information about the enemy's equipment is required in multicarrier jamming, because only some subcarriers are targeted. In simulation only fixed NLOS is considered. In OFDMA there are data subcarriers, pilot subcarriers, guard subcarriers. The simulation parameters required for multicarrier jamming are described in next section.

\section{Simulation Setup}

\subsection{Simulation Parameters for Single Carrier Jamming}

In this paper fixed NLOS WiMAX system is simulated under jamming. Single carrier jamming scenario is shown in Figure 2.

The node model of jammer is given in Figure 3.

Source parameters for the jammer are packet inter-arrival time, packet size, jammer start time, stop time and transmitter power. According to target system these parameter are chosen.

Radio parameters are data rate, packet formats, frequency and bandwidth. Frequency is chosen according to carrier frequency of target system.

Antenna can be isotropic, WiMAX Omni and WiMAX sector antenna. Polarization is also much important in practical case of jamming. If polarization of jamming system antenna is not proper then it results in wastage of power.

\subsection{Simulation Parameters for Multicarrier Jamming}

Multi carrier jamming is difficult to simulate. In this paper multi carrier jamming effect is modeled. Symbol duration is calculated by the following equation. In scalable
OFDMA the symbol duration and subcarrier spacing is

$$
\begin{aligned}
T_{s} & =T_{b}+T_{g} \\
T_{b} & =\frac{1}{\text { delta } f} \\
\text { delta } f & =\frac{\text { bandwidth }}{\text { subcarriers }} \times n \\
T_{g} & =\frac{T_{b}}{8}
\end{aligned}
$$

where, $\mathrm{n}=$ sampling factor

$$
\begin{aligned}
& \text { delta_ } f=\text { tone spacing } \\
& T_{S}=\text { symbol duration } \\
& T_{g}=\text { guard time } \\
& T_{b}=\text { useful symbol duration }
\end{aligned}
$$

fixed. Number of sub-carriers and bandwidth is changed to keep symbol duration and subcarrier spacing fixed. Equation (4) shows number of subcarriers is proportional to bandwidth of channel for scalable OFDMA. It is multicarrier scheme. Multicarrier jamming scenario is shown in Figure 4.

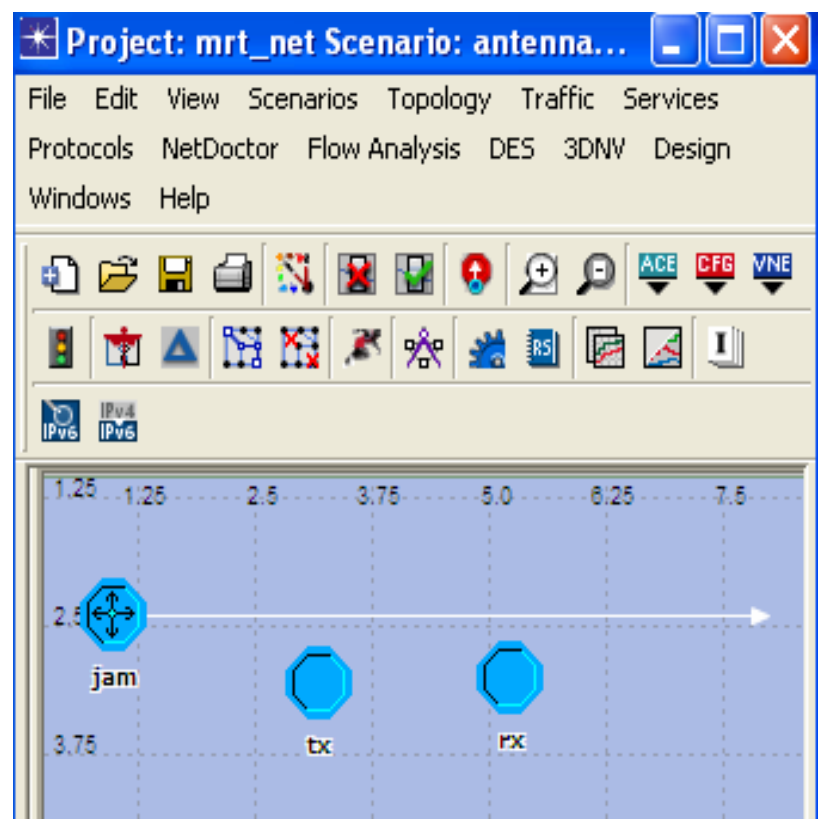

Figure 2. Scenario used for single carrier jamming

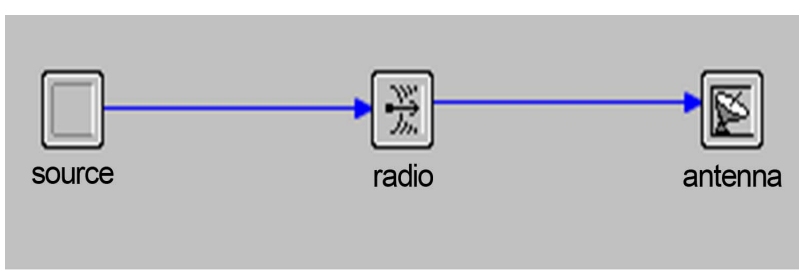

Figure 3. Node model of jammer 


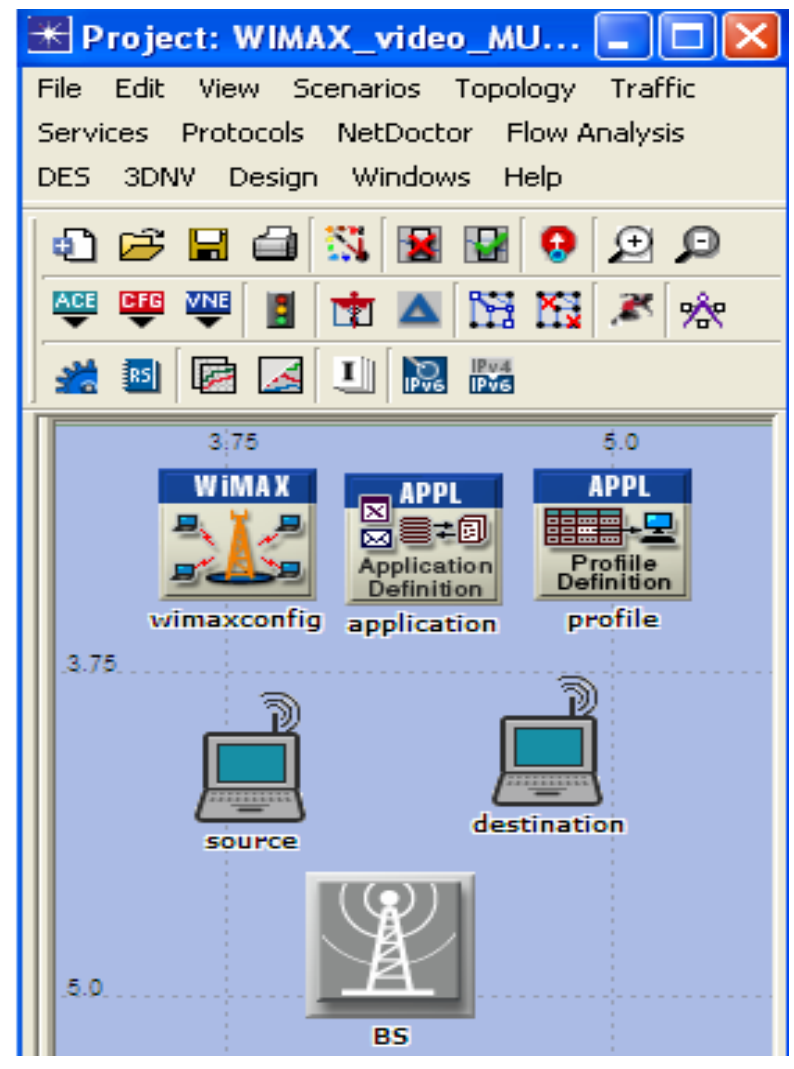

Figure 4. Scenario used to simulate multicarrier jamming

In this scenario fixed NLOS is considered. There are two subscriber stations. Application and profile node is used to create an application. WiMAX configuration node is used to configure WiMAX properties. IEEE 802.16e uses fixed and mobile NLOS. There are two subcarrier permutation modes FUSC (full usage subcarrier) and PUSC (partial usage subcarrier). Uplink and downlink both can use different permutation modes in single application $[10,11]$.

In uplink PUSC subcarrier permutation is given in Table 1 [11]. Same way in Downlink PUSC subcarrier permutation is given in Table 2. In Simulation depending on number of subcarriers and permutation scheme used parameters are chosen from the given tables.

Table 1. PUSC permutation scheme for uplink [11]

\begin{tabular}{lcc}
\hline & 512 & 1024 \\
\hline Number of sub channels & 17 & 35 \\
Data subcarriers used & 272 & 560 \\
Pilot subcarrier & 136 & 280 \\
Left-guard subcarriers & 52 & 92 \\
Right-guard subcarriers & 51 & 91 \\
\hline
\end{tabular}

Downlink PUSC permutation scheme is also used in simulation. Numbers of data, pilot and guard subcarriers required for scalable OFDMA are shown in the table.

Steps involved in multicarrier jamming simulation are described below.

1) Take the scenario as shown in Figure 4. Set the application which is to be examined under jamming effect. Select parameters for the same.

2) Now choose number of subscribers and base stations. Set their attributes according to application.

3) Set WiMAX attributes of all subscribers and base station. Set the symbol duration according to standards.

4) Set permutation mode as per the simulation criteria.

5) Now simulate the scenario and see the throughput and delay.

6) Take new scenario as shown in Figure $\mathbf{4}$ and repeat step number 1 to 4 . Reduce number of data or pilot or guard subcarriers in uplink or downlink as per simulation criteria but keep symbol duration, subcarrier spacing and bandwidth same as the first scenario.

7) Now simulate this scenario and compare the throughput and delay result with previous result.

By following above steps multicarrier jamming effect can be simulated and results can be noted down.

\section{Results}

In first scenario two subscriber stations are there and jammer is moving towards subscriber stations and then moving away as show in Figure 2. This scenario is build to simulate single carrier jamming. The simulation time is taken by considering movement of jammer. The result shown in Figure 5 is BER performance under jamming for different modulation schemes.

Second result shown in Figure 6 is taken using the same scenario but the antennas used in subscriber stations are changed to see the impact antennas under jamming.

Figure 6 shows the BER vs. simulation time for different antenna used at receiver side under jamming effect. Jammer has isotropic antenna. Now new scenario is taken as shown in Figure 4 to simulate the effect of scalability property of OFDMA. In this scenario two subscriber stations and one base station are there. Any application

Table 2. PUSC permutation scheme for downlink [1]

\begin{tabular}{lcccc}
\hline & 128 & 512 & 1024 & 2048 \\
\hline Number of sub channels & 3 & 15 & 30 & 60 \\
Data subcarriers used & 72 & 360 & 720 & 1440 \\
Pilot subcarrier & 12 & 60 & 120 & 240 \\
Left-guard subcarriers & 22 & 46 & 92 & 184 \\
Right-guard subcarriers & 21 & 45 & 91 & 183 \\
\hline
\end{tabular}




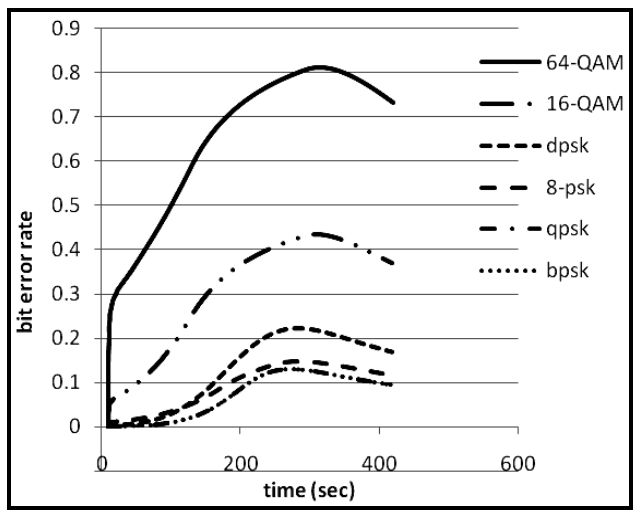

Figure 5. BER vs. simulation time

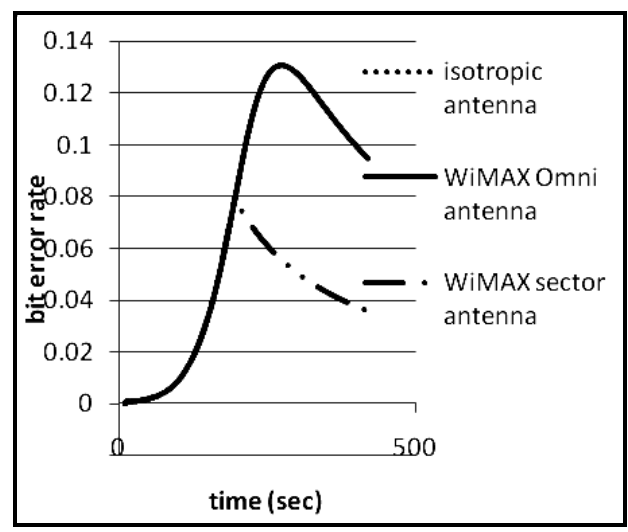

Figure 6. BER vs. simulation time

can be used to simulate multi carrier jamming. In this scenario Video conferencing application is chosen. Number of subcarriers chosen for this application is 512 and bandwidth is $5 \mathrm{MHz}$ for this application. The symbol duration is 102.86 micro seconds and subcarrier spacing is $10.94 \mathrm{KHz}$. Uplink and downlink both is using PUSC. Take new scenario as shown in Figure 4 and keep all the parameters same except number of subcarriers and bandwidth. Now choose 1024 subcarriers and $10 \mathrm{MHz}$ bandwidth. The results for scalability property are shown in Figure 7 and Figure 8. Simulation time is set to 300 seconds. Throughput and delay of whole system is considered in the results. The system throughput is the sum of all data rates that are delivered to all terminals in a network. Delay is time taken by a packet to reach its destination starting from the time it leaves the source.

Now new scenario is taken as shown in Figure 4 to simulate multicarrier jamming effect. Sampling factor $\mathrm{n}$ is 28/25 and number of subcarriers are 1024. This scenario is run for 300 seconds. Bandwidth is $10 \mathrm{MHz}$, subcarrier spacing is $10.94 \mathrm{KHz}$ same as second scenario. Data subcarriers in uplink PUSC and downlink PUSC are 560 and 720 respectively. Number of sub channels in uplink PUSC and downlink PUSC are 35 and 30 respectively.

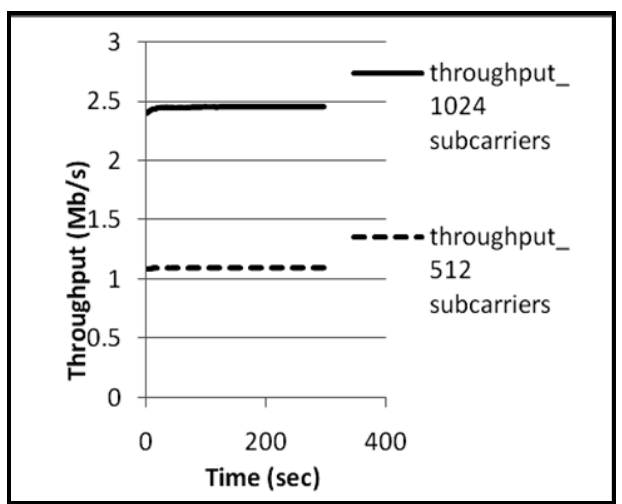

Figure 7. Throughput vs. simulation time

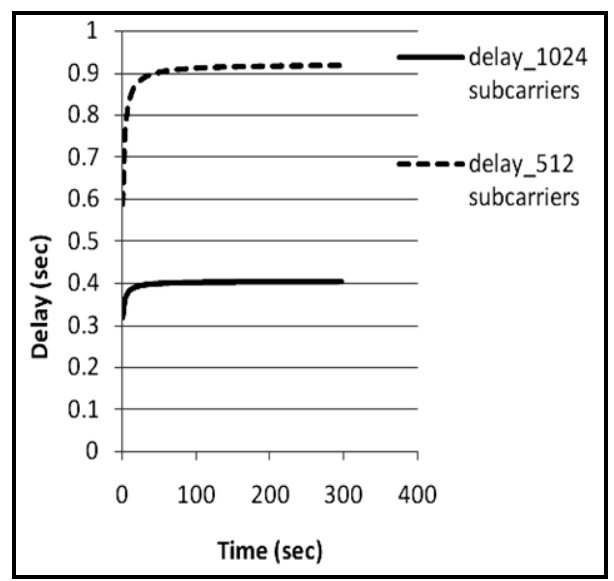

Figure 8. Delay vs. simulation time

Now follow the steps that are already mentioned in simulation section to simulate multi carrier jamming. Procedure to simulate multicarrier jamming is already mentioned. In uplink the effect of data subcarrier reduction is more compare to that in downlink. Results for uplink multicarrier jamming are shown in Figure 9 and Figure 10. Graph of throughput and delay vs. simulation time are shown.

Downlink is less sensitive to data subcarrier reduction. Here Figure 7 and Figure 8 shows the effect of scalability property of OFDMA on throughput and delay. The symbol duration and subcarrier spacing keep same for both 512 and 1024 subcarriers but the number of subcarriers and bandwidth is changed. Figure 9 and Figure 10 show the effect of number of data subcarrier reduction on throughput and delay in uplink. Throughput is decreased as numbers of data subcarriers are destroyed simulation result is shown in Figure 11. Delay is increased as the number of data subcarrier destroyed shown in Figure 12. In this paper only data subcarriers are destroyed and effect is noted down. If pilot subcarriers are destroyed then channel estimation becomes very difficult Multicarrier jamming effect on throughput and delay in downlink is shown below. 


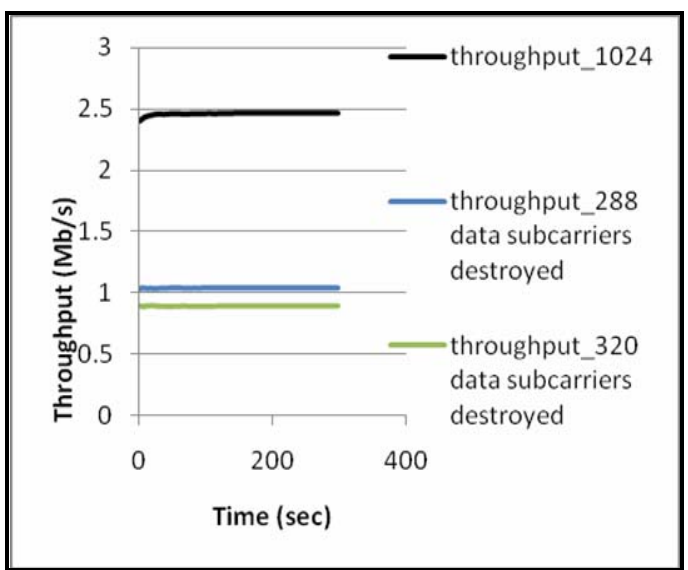

Figure 9. Throughput vs. simulation time for uplink

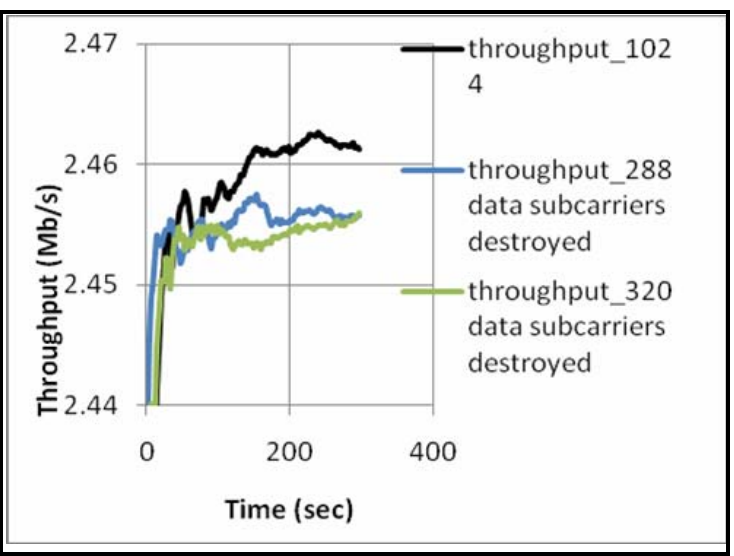

Figure 11. Throughput vs. simulation time for downlink

\section{Conclusions}

The performance of BPSK and QPSK is better than other modulation scheme under single carrier jamming. WiMAX sector antenna gives better performance than isotropic and WiMAX Omni antennas. Throughput is decreeased when data subcarriers are destroyed in uplink and downlink. Throughput is reduced means BER is increased. Delay is increased when data subcarriers are destroyed in uplink and downlink. Uplink is more sensitive to data subcarrier reduction compare to downlink. Single carrier jamming and multicarrier jamming both are destructive in nature. Single carrier jamming can be detected easily compare to multicarrier jamming. The performance of the downlink PUSC is better than uplink PUSC under the influence of the multicarrier jamming. Multicarrier jamming effects are modeled in this paper by following particular algorithm which is already mentioned earlier. In this paper only simulated results are shown. In practical multicarrier jamming effect on throughput and delay remains same as shown by the simulated results. In practical case of multicarrier jamming antenna polariza-

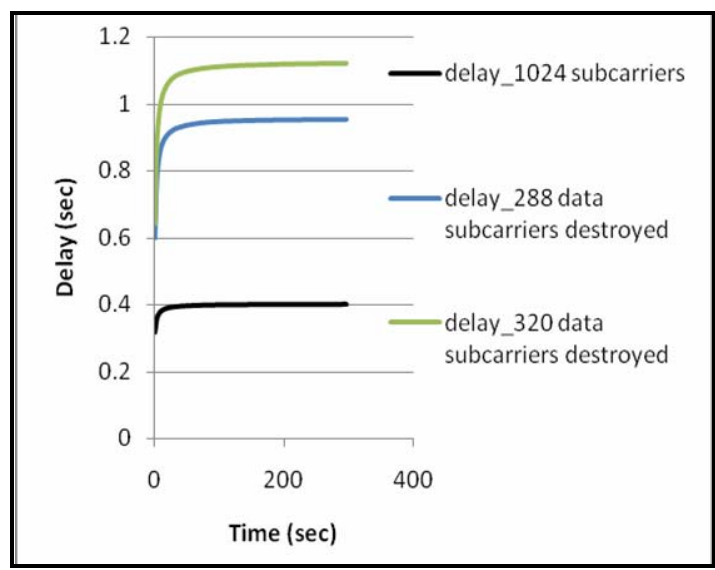

Figure 10. Delay vs. simulation time for uplink

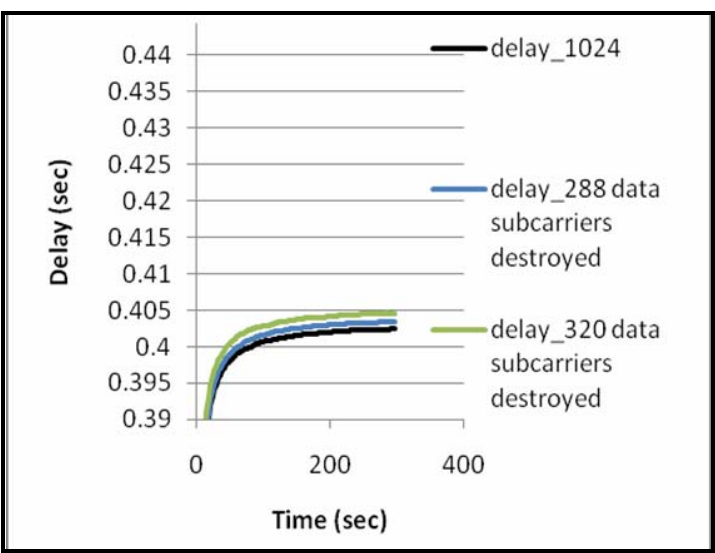

Figure 12. Delay vs. simulation time for downlink

tion and its pattern have to be considered. The paper shows results for fixed NLOS only. Mobile NLOS can be considered with some additional simulation parameters related to mobility under jamming effect.

\section{REFERENCES}

[1] J. G. Andrews, A. Ghosh and R. Muhamed, "Fundamentals of WiMAX Understanding Broadband Wireless Networking,” Prentice-Hall, Upper Saddle River, 2007.

[2] S. Haykin and M. Moher, "Modern Wireless Communication,” Prentice-Hall, Upper Saddle River, 2005.

[3] S. Ahson, M. Ilyas, S. Ahson and M. Ilyas, "WiMAX Standards and Security,” CRC Press, Boca Raton, 2008.

[4] T. Shon and W. Choi, "An Analysis of Mobile WiMAX security: Vulnerabilities and Solutions," Lecture Notes in Computer Science, Springer Berlin, Vol. 4658, 2007, pp. 88-97.

[5] C. L. Luo, "A Simple Encryption Scheme Based on WiMAX,” Department of Electronics Jiangxi University of Finance and Economics Nanchang, China, 2009.

[6] B. Makarevitch, "Jamming Resistant Architecture for 
WiMAX Mesh Network,” Communications Laboratory, Helsinki University of Technology, Helsinki, June 2006.

[7] J. Li and S.-G. Häggman, "Performance of IEEE 802.16 Based System in Jamming Enviornment and its Improvement with Link Adaption,” The 17th Annual IEEE International Symposium on Personal, Indoor and Mobile Radio Communications (PIMRC’06), 2006, Helsinki, pp. 1-5.

[8] M. Nasreldin, H. Aslan, M. El-Hennawy and A. El-Hennawy, "WiMAX Security," 22nd International Conference on Advanced Information Networking and applications, Ginowan, 2008, pp. 1335-1340.

[9] White Paper by Motorola, "WiMAX Security for Real-
World Network Service Provider Deployments,” 2007.

[10] IEEE 802.16 Working Group, “IEEE 802.16-2004 Local and Metropolitan Area Networks - Part 16: Air Interface for Fixed Broadband Wireless Access Systems IEEE Standard for Local and Metropolitan Area Networks," IEEE Computer Society Press, 2004.

[11] IEEE 802.16 Working Group, “IEEE 802.16e-2005 IEEE Standard for Local and Metropolitan Area Networks Part 16: Air Interface for Fixed and Mobile Broadband Wireless Access Systems Amendment for Physical and Medium Access Control Layers for Combined Fixed and Mobile Operation in Licensed Bands," IEEE Computer Society Press, 2005. 